\title{
Neuroprotective effects of resveratrol against traumatic brain injury in rats: Involvement of synaptic proteins and neuronal autophagy
}

\author{
YAN FENG ${ }^{1}$, YING CUI ${ }^{2}$, JUN-LING GAO ${ }^{3,4}$, RAN LI $^{3,4}$, XIAO-HUA JIANG ${ }^{3,4}$, YAN-XIA TIAN ${ }^{3,4}$, \\ KAI-JIE WANG ${ }^{2}$, MING-HANG LI ${ }^{3}$, HONG-AO ZHANG ${ }^{3}$ and JIAN-ZHONG CUI ${ }^{2}$ \\ ${ }^{1}$ Department of Neurosurgery, Second Hospital of Hebei Medical University, Shijiazhuang, Hebei 050000; \\ ${ }^{2}$ Department of Neurosurgery, Tangshan Workers' Hospital; ${ }^{3}$ School of Basic Medical Science, \\ North China University of Science and Technology; ${ }^{4}$ Hebei Key Laboratory for Chronic Diseases; \\ Tangshan Key Laboratory for Preclinical and Basic Research on Chronic Diseases, \\ North China University of Science and Technology, Tangshan, Hebei 063000, P.R. China
}

Received April 26, 2015; Accepted March 29, 2016

DOI: $10.3892 / \mathrm{mmr} .2016 .5201$

\begin{abstract}
Traumatic brain injury (TBI) involves primary and secondary injury cascades that underlie delayed neuronal dysfunction and death, leading to long-term cognitive deficits, and effective therapeutic strategies targeting neuronal death remain elusive. The present study aimed to determine whether the administration of resveratrol $(100 \mathrm{mg} / \mathrm{kg})$ was able to significantly enhance functional recovery in a rat model of TBI and whether resveratrol treatment was able to upregulate synaptic protein expression and suppress post-TBI neuronal autophagy. The results demonstrated that daily treatment with resveratrol attenuated TBI-induced brain edema and improved spatial cognitive function and neurological impairment in rats. The expression of synaptic proteins was downregulated following TBI and this phenomenon was partly reversed by treatment with resveratrol. In addition, resveratrol was observed to significantly reduce the levels of the autophagic marker proteins, microtubule-associated protein light chain 3-II and Beclin1, in the hippocampus compared with the TBI group. Therefore, these results suggest that resveratrol may represent a novel therapeutic strategy for TBI, and that this protection may be associated with the upregulation
\end{abstract}

Correspondence to: Dr Jian-Zhong Cui, Department of Neurosurgery, Tangshan Workers' Hospital, 27 Wen Hua Road, Tangshan, Hebei 063000, P.R. China

E-mail: jzhcuidr2001@163.com

Abbreviations: RV, resveratrol; TBI, traumatic brain injury; SYN, synaptophysin; PSD95, post synaptic density 95; LC3, microtubule-associated protein 1 light chain 3; NeuN, neuron-specific nuclear protein; DAPI, 4',6-diamidino-2-phenylindole; NSS, neurologic severity score

Key words: neuroprotective, resveratrol, traumatic brain injury, synaptic proteins, autophagy of synaptophysin, postsynaptic density protein 95 and the suppression of neuronal autophagy.

\section{Introduction}

Traumatic brain injury (TBI) is a leading cause of mortality and disability in young individuals and represents an important public health issue worldwide. Primary and secondary injury cascades resulting in delayed neuronal dysfunction, synapse loss and cell death are associated with TBI $(1,2)$. However, the precise mechanisms underlying the secondary injury are not well understood, and one result of TBI is long-term cognitive dysfunction and memory loss, which affects all patients with TBI. A number of studies have demonstrated that TBI leads to synaptic loss $(1,3)$, or disrupts synaptic plasticity (4), which contributes to long-term behavioral disorders. However, the molecular mechanisms by which TBI causes synapse loss in the early stages remain unclear.

Autophagy is an evolutionarily conserved pathway that results in the degradation of proteins and entire organelles in cells undergoing stress (5). Although autophagy constitutes a stress adaptation pathway that promotes cell survival under the majority of circumstances, increasingly studies have demonstrated that it may trigger cell injury and death under certain pathological circumstances $(6,7)$. Previous studies have demonstrated that the autophagic pathway is involved in the pathophysiological response following TBI, and inhibition of this pathway may help to attenuate traumatic damage and functional deficits $(8-10)$.

Resveratrol (3,5,40-trihydroxystilbene, RV) occurs naturally in grapes and a variety of medicinal plants, and exhibits multiple biological activities $(11,12)$. In particular, RV exerts protective effects against neurological damage in a number of animal models, including stroke, spinal cord injury and neurodegenerative diseases (13-15). The beneficial effects of RV in central nervous system injuries are associated with its anti-oxidant (16), anti-inflammatory (17) and anti-apoptotic properties (18). However, the protective 
effect and mechanisms of RV treatment following TBI require further examination.

In the current study, the effect of RV on post-TBI brain edema, spatial cognitive function and neurological impairment was examined in a rat model. Synaptic proteins and neuronal autophagy were also assessed. The results may provide evidence of $\mathrm{RV}$-mediated neuroprotection in a rat model of TBI.

\section{Materials and methods}

Animals and TBI model. All experimental procedures were conducted in accordance with the guidelines of the Chinese council on animal protection (http://www.hebstd.gov. cn/banshi/zxyw/content_74239.htm) and were approved by the Hebei Medical University (Shijiazhuang, China) Committee for the use of animals in research. A total of 150 adult (age, 12-16 weeks) male Sprague-Dawley rats weighing 300-330 g were obtained from Hebei United University Experimental Animal Center (Tangshan, China). The animals were maintained at $21-26^{\circ} \mathrm{C}$ under a 12 -h light/dark cycle, and water and food were provided ad libitum prior to and following surgery or sham surgery. The rat model of TBI was induced using a weight-drop device, as previously described by Marmarou et al (19). Briefly, the rats were anesthetized with $10 \%$ chloral hydrate $(3 \mathrm{ml} / \mathrm{kg})$. A midline incision was made to expose the skull between the bregma and lambda suture lines and a steel disc ( $10 \mathrm{~mm}$ in diameter and $3 \mathrm{~mm}$ thickness) was adhered to the skull using dental acrylic. Then rats were placed on a foam mattress underneath a weight-drop device in which a $350 \mathrm{~g}$ weight falls freely through a vertical tube from $1.5 \mathrm{~m}$ onto the steel disk. Following injury, the scalp was sutured. Rats were housed in individual cages and placed on heat pads $\left(37^{\circ} \mathrm{C}\right)$ for $24 \mathrm{~h}$ to maintain normal body temperature during the recovery period. This model is generally associated with $20 \%$ mortality within the first 5 min post-injury and no delayed mortality was observed thereafter. The sham-operated animals were anesthetized and had the steel disk attached to them, but they did not receive TBI.

Groups and drug administration. Rats were randomly divided into three groups: Sham operation group (sham, n=30); TBI group (TBI, $n=60$ ); and TBI treated with RV group (RV, $\mathrm{n}=60$ ). RV (Sigma-Aldrich, St. Louis, MO, USA) was freshly prepared by dissolving in $50 \%$ ethanol and diluting in physiological saline (2\%) at a concentration of $100 \mathrm{mg} / \mathrm{kg}$, and was administered daily via intraperitoneal injection to the rats in the RV group for 5 days beginning immediately following TBI (14). Both the sham and TBI groups received equal volumes of ethanol $(2 \%)$ by intraperitoneal injection at the same time daily. Each subgroup was composed of five rats and the rats were sacrificed at 1,3 and 5 days following TBI.

Evaluation of brain edema. Brain edema was evaluated by analysis of brain water content using the wet-dry weight method, as described previously (20). Briefly, rats were randomly sampled from each group and anesthetized by intraperitoneal injection with $10 \%$ chloral hydrate $(3 \mathrm{ml} / \mathrm{kg} ; \mathrm{n}=5)$. The cerebral tissues were then removed and weighed immediately on an electric analytic balance for the wet weight, then dried at $100^{\circ} \mathrm{C}$ for $24 \mathrm{~h}$ to obtain the dry weight. The percentage of water in the tissues was calculated according to the formula: $\%$ brain water $=[($ wet weight - dry weight $) /$ wet weight $] \times 100$.

Morris water maze (MWM) test. To evaluate spatial learning and memory, rats were tested in variation of the MWM para$\operatorname{digm}(21)$. The maze consists of a water-filled pool $(180 \mathrm{~cm}$ diameter, $45 \mathrm{~cm}$ high) at $26^{\circ} \mathrm{C}$ and virtually divided into 4 equivalent quadrants: North $(\mathrm{N})$, west $(\mathrm{W})$, south $(\mathrm{S})$ and east (E). A $2 \mathrm{~cm}$ submerged escape platform (diameter $12 \mathrm{~cm}$, made opaque with paint) was placed in the middle of one of the quadrants equidistant from the sidewall and the center of the pool. All experimental rats were trained to find the platform prior to TBI or sham surgery. At the start of a trial, the rat was placed at one of four fixed starting points, randomly facing a wall (designated N, S, E and W) and allowed to swim for $60 \mathrm{sec}$ or until it found the platform. If the animal found the platform, it was allowed to remain on it for $20 \mathrm{sec}$. If the animal failed to find the platform within $90 \mathrm{sec}$, it was placed on the platform for $20 \mathrm{sec}$. The time required (escape latency) to find the hidden platform with a $60 \mathrm{sec}$ limit was recorded by a video camera suspended above the maze and interfaced with a video tracking system (HVS Imaging, Hampton, UK). The test was conducted at 3-5 days following TBI or sham surgery, and each rat was tested for four trials per day for three consecutive days. The average escape latency of the total of the four trials was calculated.

Modified neurological severity score. Neurological deficits were evaluated using the Neurologic Severity Score (NSS) on a 10-point scale, as previously described by Chen et al (22) by a researcher blinded to treatment. One point is awarded for failure to perform a particular task, and a score of ten reflects maximal impairment, thus, a normal rat scores zero. Post-injury, the NSS was evaluated at days 1-5.

Western blot analysis. Western blotting was performed as previously described (23). At scheduled time points, rats were anesthetized with $10 \%$ chloral hydrate and decapitated. The brains were quickly removed and the hippocampal tissues were dissected on ice. Total proteins were extracted and the protein concentration was determined using a bicinchoninic acid kit (Beijing Solarbio Science \& Technology Co., Ltd., Beijing, China). Equal amounts of protein $(50 \mathrm{mg})$ was subjected to $10 \%$ sodium dodecyl sulfate-polyacrylamide gel electrophoresis (Beyotime Institute of Biotechnology, Haimen, China). Separated proteins on the gel were transferred onto polyvinylidene fluoride membranes (Roche Diagnostics $\mathrm{GmbH}$, Mannheim, Germany) by a transfer apparatus at $200 \mathrm{~mA}$ for $50 \mathrm{~min}$. The membrane was then blocked with $5 \%$ fat-free dry milk for $2 \mathrm{~h}$ at room temperature. Subsequently, blots were incubated with the following primary antibodies overnight at $4^{\circ} \mathrm{C}$ : Polyclonal rabbit anti-microtubule-associated protein light chain 3 (LC3) [cat. no. PD014; Molecular \& Biological Laboratories Co., Ltd., Nagoya, Japan (dilution, 1:1,000)], polyclonal rabbit anti-Beclin1 [cat. no. JM-3663100; Molecular \& Biological Laboratories Co., Ltd. (dilution, 1:1,000)], polyclonal rabbit anti-p62 [cat. no. ARH4210; Antibody Revolution, Inc. San Diego, CA, USA (dilution, 1:1,000)], monoclonal rabbit anti-postsynaptic density 
protein 95 (PSD95) [cat. no. ab76108; Abcam, Cambridge, UK (dilution,1:500)], monoclonal rabbit anti-synaptophysin (SYN) [cat. no. ARH4007; Antibody Revolution, Inc. (dilution, 1:500)], polyclonal rabbit anti- $\beta$-actin [cat. no. AF7018; Affinity Biologicals, Inc., Cincinnati, OH, USA (dilution, 1:1,000)]. The membranes were then incubated with horseradish peroxidase-conjugated anti-rabbit IgG [cat. no. sc-2004; Santa Cruz Biotechnology, Inc., Dallas, TX, USA (dilution, $1: 5,000)]$ for $2 \mathrm{~h}$ at room temperature, prior to development using an enhanced chemiluminescence detection system [MultiSciences (LIANKE) Biotech Co., Ltd., Hangzhou, China] and the densitometric signals were quantified using an imaging program (Image Lab 4.1; Bio-Rad Laboratories, Inc., Hercules, CA, USA). The immunoreactive bands were normalized to rabbit anti- $\beta$-actin polyclonal antibody (dilution, 1:1,000). Results were analyzed using the National Institutes of Health ImageJ 1.41 software (Bethesda, MD, USA).

Immunofluorescence analyses. Rats were perfused transcardially with saline under deep anesthesia [10\% chloral hydrate $(3 \mathrm{ml} / \mathrm{kg})$ by intraperitoneal injection]. The brain tissues were the fixed using $4 \%$ formaldehyde for $24 \mathrm{~h}$, transferred to a $30 \%$ sucrose solution [0.1 M phosphate-buffered saline (PBS), $\mathrm{pH}$ 7.4] until they sank to the bottom, following which they were embedded in optimal cutting temperature compound. The brains were cut into $15 \mu \mathrm{m}$-thick sections coronally from the anterior to posterior hippocampus (bregma -2.0 to $-3.5 \mathrm{~mm}$ ) using a cryostat. Frozen sections were treated with $0.4 \%$ Triton X-100 for $30 \mathrm{~min}$, and blocked in normal donkey serum for $1 \mathrm{~h}$. For double labeling, the frozen sections were incubated with a mixture of polyclonal rabbit anti-LC3 (1:100) and monoclonal mouse anti-NeuN [cat. no. MAB377; EMD Millipore, Billerica, MA, USA (dilution, 1:100)] antibodies overnight at $4^{\circ} \mathrm{C}$. The following day, the sections were incubated with a mixture of donkey anti-rabbit IgG-fluorescein isothiocyanate (FITC) and donkey anti-mouse IgG-FITC [cat. nos. sc-2090 and sc-2099, respectively; Santa Cruz Biotechnology, Inc. (dilution, 1:1,000)] for $2 \mathrm{~h}$ at $37^{\circ} \mathrm{C}$ in the dark. All cell nuclei were counterstained by 4',6-diamidino-2-phenylindole (DAPI). Images were captured using a laser scanning confocal microscope (Olympus Fluoview $^{\mathrm{TM}}$ FV1000; Olympus Corporation, Tokyo Japan). Primary antibodies were replaced with PBS in the negative control group.

Statistical analysis. Statistical analysis was performed using SPSS software, version 16.0 (SPSS, Chicago, IL, USA). Data are presented as the mean \pm standard deviation. Statistical analysis was performed using one-way analysis of variance followed by the Student-Newman-Keuls post hoc multiple comparisons test. $\mathrm{P}<0.05$ was considered to indicate a statistically significant difference.

\section{Results}

$R V$ treatment attenuates brain edema. The wet-dry weight method was used to evaluate brain edema. As shown in Fig. 1, TBI induced a significant increase in brain edema at 1, 3 and 5 days in the TBI group compared with the sham control. RV administered post-injury significantly reduced brain edema following TBI.

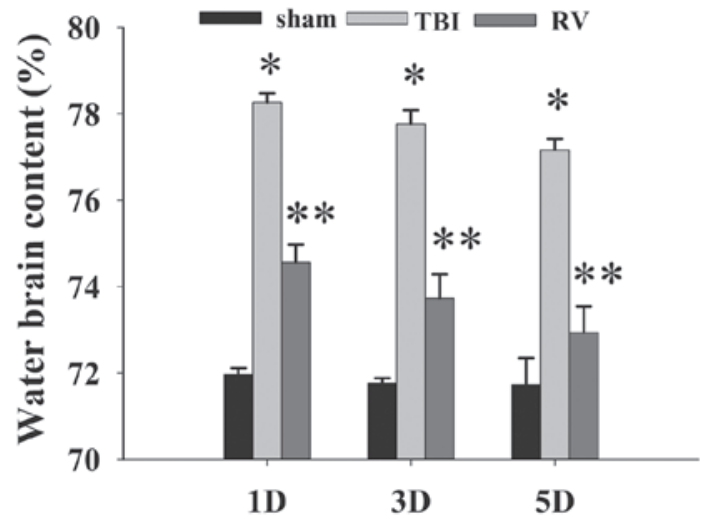

Figure 1. The effect of RV on brain edema. Brain water content increased markedly at 1,3 and 5 days following TBI . Treatment with RV significantly reduced brain edema. Data are presented as the mean \pm standard deviation $(n=5) . ~{ }^{*} \mathrm{P}<0.01$ vs. sham group; ${ }^{* *} \mathrm{P}<0.05$ vs. TBI group. $\mathrm{RV}$, resveratrol; TBI, traumatic brain injury; D, days.

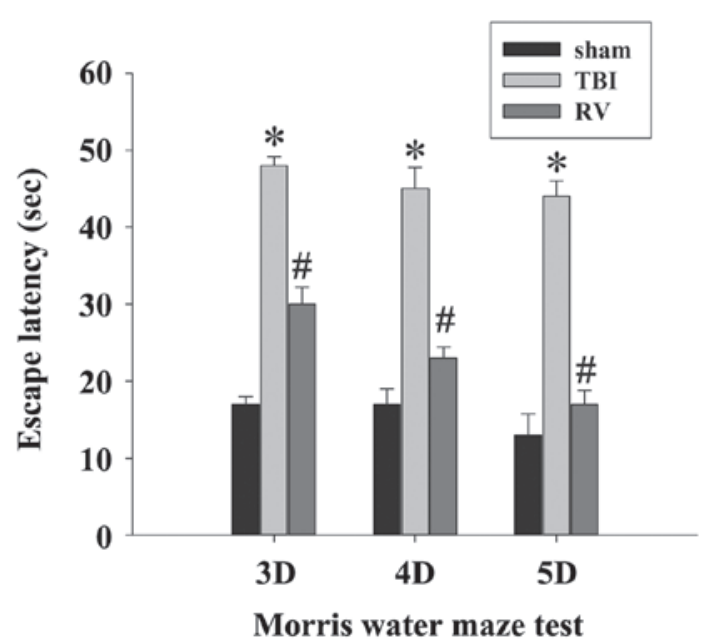

Figure 2. The effect of RV on the escape latency. The escape latency was markedly increased and treatment with RV significantly reduced the time to find the platform at 3,4 and 5 days following injury. Data are presented as the mean \pm standard deviation $(\mathrm{n}=5)$. ${ }^{*} \mathrm{P}<0.01$ vs. sham group; ${ }^{\text {"P }} \mathrm{P}<0.05$ vs. TBI group. RV, resveratrol; TBI, traumatic brain injury; D, days.

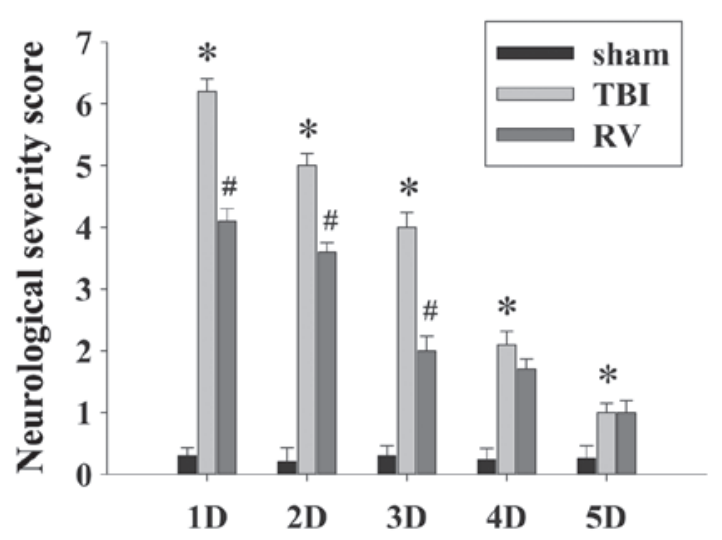

Figure 3. The effect of RV on NSS score. The NSS of rats in the TBI group were significantly increased in comparison with the sham group at 1-4 days and the administration of RV significantly improved motor function at 1-3 days following TBI. " $\mathrm{P}<0.01$ vs. sham group; ${ }^{\#} \mathrm{P}<0.05$ vs. TBI group. $\mathrm{RV}$, resveratrol; NSS, neurologic severity score; TBI, traumatic brain injury; D, days. 
A

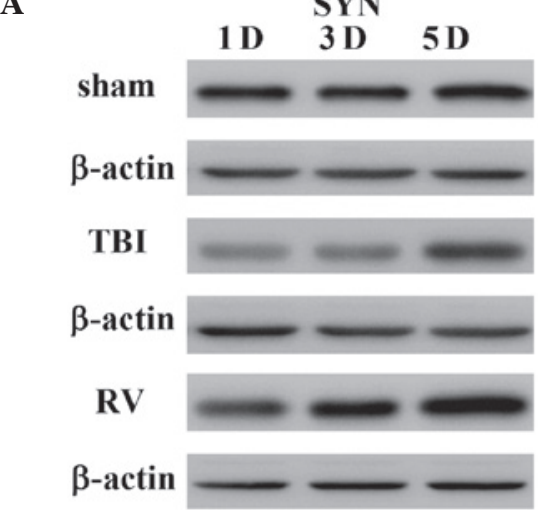

B

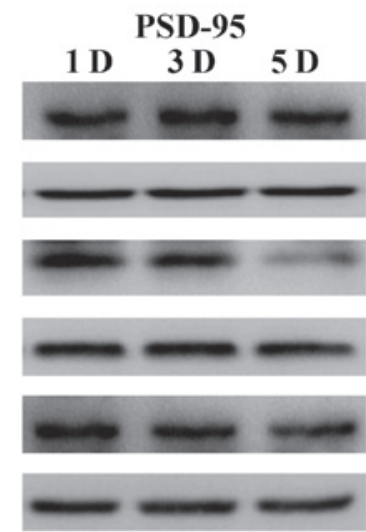

C

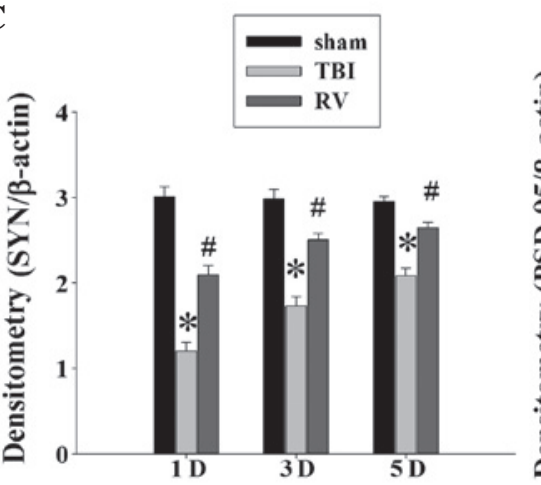

D

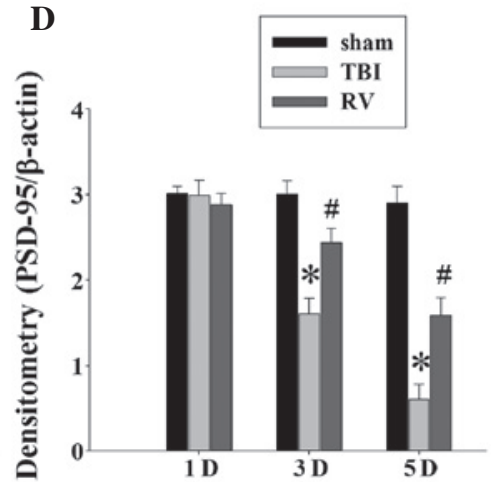

Figure 4. Effect of RV on the expression of synaptic proteins. Western blot images showing the expression of (A) SYN and (B) PSD-95 in the hippocampi of rats at 1, 3 and 5 days following TBI or sham surgery. Densitometry analysis of (C) SYN and (D) PSD-95 bands relative to $\beta$-actin. This demonstrated a significant downregulation of SYN expression at 1, 3 and 5 days in the TBI group. Treatment with RV resulted in a significant increased in SYN expression levels. PSD-95 protein levels were markedly reduced at 3 and 5 days following TBI, with RV treatment resulting in a significant increase compared with the TBI group. Data are presented as the mean \pm standard deviation $(\mathrm{n}=5)$. ${ }^{*} \mathrm{P}<0.01$ vs. sham group; ${ }^{\#} \mathrm{P}<0.05$ vs. TBI group. RV, resveratrol; $\mathrm{SYN}$, synaptophysin; PSD-95, postsynaptic density protein 95; TBI, traumatic brain injury; D, days.

$R V$ treatment improves learning and memory ability and motor deficits. To determine the neuroprotective effects of $\mathrm{RV}$ against TBI-induced brain damage, the effects of RV pretreatment on the learning and memory ability (Fig. 2) and motor deficits (Fig. 3) were examined using the MWM and NSS score following TBI, respectively. As expected, TBI resulted in a significant deficit in spatial learning compared with the sham group, and the administration of RV significantly reduced the escape latency at 3,4 and 5 days compared with the TBI group. In addition, the NSS of the rats in the TBI group were observed to significantly increase in comparison with the sham group at 1-4 days, and RV treatment significantly improved the motor function recovery of the TBI rats at 1-3 days following injury.

$R V$ treatment attenuates synaptic protein loss in the hippocampus. A number of studies have reported the significant loss of synapses in the days following brain injury, including in the brain regions connected to the site of initial injury, such as the hippocampus $(1,4,24)$. Considering RV was able to improve spatial learning, it was examined whether RV treatment could affect synaptic alterations in the rat hippocampus following TBI. The expression of two synaptic proteins was evaluated in the rat hippocampus using western blot analysis at 1,3 and 5 days following TBI. As demonstrated in Fig. 4A, there was a reduction in the expression levels of synaptophysin in the TBI group compared with the sham group at 1, 3 and 5 days.
The reduction in the levels of synaptophysin indicated a loss of synapses in the TBI rats. The rats treated with RV exhibited significantly greater levels compared with the TBI group. In addition, Fig. 4B indicates that the levels of PSD-95 were reduced at 3 and 5 days following injury, with the RV treated rats significantly improved compared with the TBI group.

$R V$ treatment suppresses neuronal autophagy in the hippocampus. To determine how autophagic activity is altered following TBI, LC3 expression in hippocampal neurons at $24 \mathrm{~h}$ following TBI was detected using immunofluorescence. As shown in Fig. 5, LC3 staining is indicated in red, neurons stained with NeuN are in green, and DAPI staining of nuclei is in blue. The images were merged, and the co-localization of NeuN and LC3 was observed in the immunofluorescent staining at $24 \mathrm{~h}$. The results indicated that alterations in LC3 proteins had occurred in neurons in the hippocampal region following TBI. In order to confirm the ability of RV to inhibit autophagy, the protein levels of LC3-II (ratio of LC3-II to LC3-I) and Beclin1 were determined by western blot analysis. The protein expression levels of LC3-II and Beclin1 in the hippocampus were significantly upregulated at 1,3 and 5 days following TBI, with the highest level observed at 1 day (Fig. 6A). As the densitometry analysis indicates in Fig. 6B, treatment with RV significantly reduced the relative protein expression of LC3-II and Beclin1 at 1,3 and 5 days in the hippocampus compared with the TBI group. 


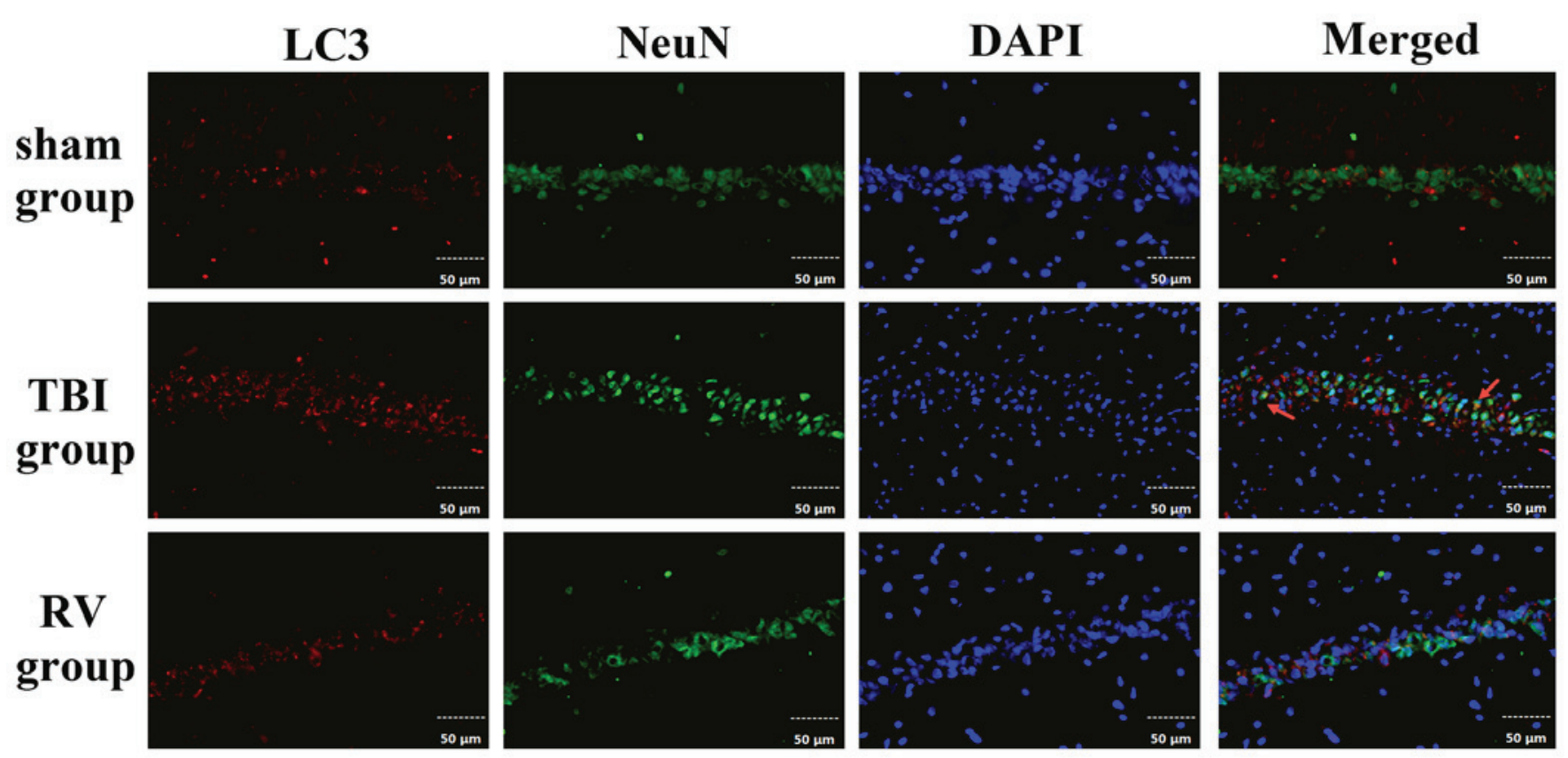

Figure 5. Co-localization of LC3 and NeuN were determined by immunofluorescent staining at $24 \mathrm{~h}$ in the sham, TBI and RV groups (scale bar, $50 \mu \mathrm{m}$ ). The orange coloring in the merged image is indicative of co-localization (red arrows). Magnification, x200. LC3, microtubule-associated protein light chain 3; DAPI, 4',6-diamidino-2-phenylindole; TBI, traumatic brain injury; RV, resveratrol.

A
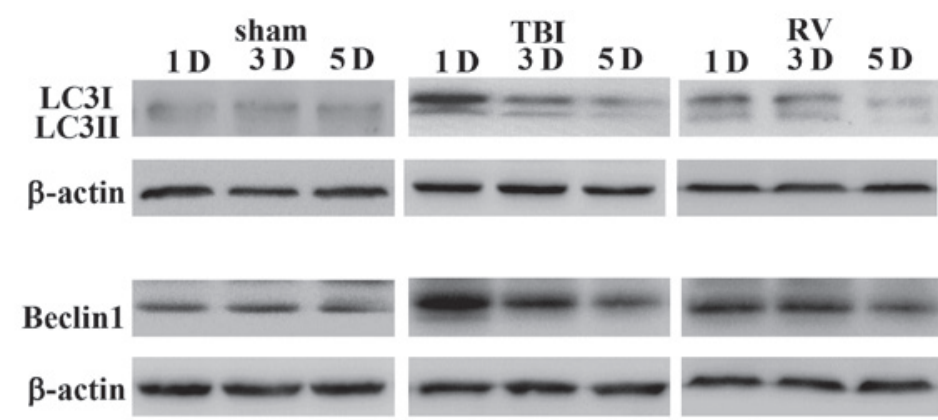

B
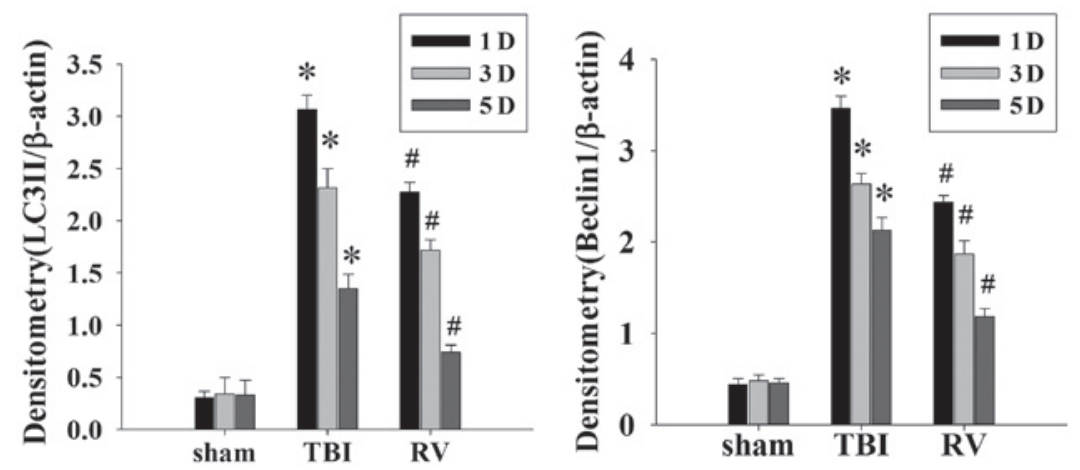

Figure 6. Effect of RV on the expression of autophagy-related proteins. (A) Western blot image showing the levels of LC3II, Beclin1 and $\beta$-actin in the hippocampus at 1,3 and 5 days following TBI or sham surgery. (B) Densitometry analysis of LC3II and Beclin1 relative to $\beta$-actin. Results demonstrated that LC3II and Beclin1 protein expression was significantly increased at 1,3 and 5 days following TBI. Administration of RV significantly reduced the protein expression levels of LC3II and Beclin1. Data are presented as the mean \pm standard deviation $(\mathrm{n}=5)$. ${ }^{*} \mathrm{P}<0.01$ vs. sham group; ${ }^{*} \mathrm{P}<0.05$ vs. TBI group. RV, resveratrol; LC3; microtubule-associated protein light chain 3; TBI, traumatic brain injury; D, days.

\section{Discussion}

Traumatic brain injury is characterized by neuronal damage and commonly, secondary cell death, leading to neurological dysfunction. The loss of neurons in the hippocampus contributes to the impairment of learning and memory following TBI.
Thus, impairment of cognitive function has long been thought to be the result of rapid cell death following TBI in humans. As a therapeutic strategy, use of safe nutritional supplements may be promising for reducing brain damage and staving off long-term cognitive deficits as observed in instances of TBI. With respect to the latter, the nutritional supplement, $\mathrm{RV}$, is a 
promising therapy to treat secondary brain injury following TBI. RV has been previously reported to provide neuroprotection against secondary brain injury, through its antioxidant and anti-inflammatory activity $(16,25)$, reducing neuronal death and glial activation (26), reducing hippocampal degeneration and improving cognitive performance (27). However, the specific mechanisms by which RV exerts its neuroprotective activity remain unclear.

In the present study, the neuroprotective potential of RV was investigated in a rat model of TBI. Rats treated with RV (100 mg/kg/day, up to 5 days) exhibited reduced posttraumatic brain edema and improved learning and memory ability and motor deficits. These results are similar to previous studies, which reported that $\mathrm{RV}$ treatment resulted in neuroprotection against a variety of neurologic disorders, in particular, acute brain injury (27). In addition, the current study demonstrates that RV was observed to protect synaptic proteins in the hippocampus. TBI leads to a decline in both synaptophysin and PSD-95, and treatment with RV showed significantly greater proteins levels compared with the TBI group, with protein levels peaking at 5 days. This is the first report, to the best of our knowledge, that RV can protect key synaptic proteins following TBI.

Synaptophysin is a $38-\mathrm{kDa}$ calcium-binding glycoprotein found in the membranes of presynaptic vesicles in neurons and is involved in vesicular trafficking, docking, synaptogenensis, synaptic reorganization and vesicular fusion with the synaptic plasma membrane (28). It has been reported to be expressed in presynaptic vesicles of the brain, spinal cord and retina, in addition to at neuromuscular junctions. Furthermore, synaptophysin has been widely used as a marker protein to quantify the number of synapses during neuroanatomical remodeling or following injury $(29,30)$. In the current study, synaptophysin was used to measure synapse loss following TBI. TBI was observed to result in synapse loss or damage, and may contribute to the observed behavioral, cognitive and neurological deficits. These results are supported by previous reports that TBI causes significant synapse loss in the hippocampal CA1 region at 2 days post-injury by $60 \%$ (1). In addition, Ding et al (31) quantified the synaptic loss following closed TBI in rats, and observed that a diffuse brain injury achieved by the use of the Marmarou model resulted in synaptic damage and reduced synaptophysin expression at both the transcriptional and translational levels (31). PSD-95, a scaffolding protein that is abundantly expressed within excitatory synapses, has been implicated in various important roles in the regulation of ion-channel function, neuronal differentiation, synaptogenesis, synaptic plasticity, and the processes of learning and memory $(32,33)$. Western blotting of PSD-95 was performed to determine the effect of TBI on the number of synapses in the hippocampus. In the present study, the PSD-95 levels were markedly reduced in the hippocampus at 3 and 5 days following TBI. A previous study indicated that PSD-95 follows a time-dependent reduction following TBI in rats. Following controlled cortical impact, using to model moderate TBI in rats, a marked reduction in PSD-95 levels in the hippocampus was observed at day 3-7 following TBI (34). The loss of PSD-95 has been directly correlated with a reduction in cognitive function in rats, suggesting a possible cellular mechanism that may, at least in part, explain the neurological deficits observed weeks and even months following the initial trauma (35). The present study observed that RV was able to increase the levels of both pre- and post-synaptic proteins in the hippocampus, which may explain why RV improved learning and memory ability, and may indicate it to be a promising treatment. Further studies are required to clarify the physiological consequences of the hippocampal protection.

Furthermore, it is worth noting that the present study observed that following injury, administration of RV was able to suppress neuronal autophagy in the rat hippocampus. Accumulating evidence suggests that the autophagic pathway is involved in the pathophysiological response to TBI, and that the inhibition of this pathway may help attenuate traumatic damage and functional outcome deficits $(8-10,23,36)$. Lin et al (18) reported that RV can increase cell survival by suppressing glycogen synthase kinase-3-mediated autophagy and apoptosis using in vivo and in vitro TBI models. Another previous study demonstrated that the combination of rapamycin and RV blocked rapamycin-induced autophagy, however, promoted apoptosis in tuberous sclerosis complex 2-deficient cells (37). In the present study, the results are in agreement with previous studies, and therefore it may be hypothesized that the neuroprotective effects of RV on TBI may be associated with the attenuation of neuronal autophagy, which is a contributing factor to neuronal death.

In conclusion, the results of the current study demonstrated that the post-injury administration of RV was able to attenuate brain edema and enhance cognitive functional recovery. In addition, RV treatment resulted in a marked elevation of synaptic proteins and suppressed neuronal autophagy induced following TBI in rats. It remains to be investigated whether $\mathrm{RV}$ is able to provide significant neuroprotection when administration is delayed following injury. It is additionally unclear whether the current dosage and route of administration of RV provides the maximal neuroprotective benefit. Therefore, further investigations are required to improve the understanding of the neuroprotective effects of RV in TBI. The results of the present study suggest that RV is a potential therapeutic compound and may provide novel clinical efficacy for the treatment of TBI.

\section{Acknowledgements}

The present study was supported by a grant from the Natural Science Foundation of Hebei Province (grant no. H2014105079).

\section{References}

1. Scheff SW, Price DA, Hicks RR, Baldwin SA, Robinson S and Brackney C: Synaptogenesis in the hippocampal CA1 field following traumatic brain injury. J Neurotrauma 22: 719-732, 2005.

2. Baldwin SA, Gibson T, Callihan CT, Sullivan PG, Palmer E and Scheff SW: Neuronal cell loss in the CA3 subfield of the hippocampus following cortical contusion utilizing the optical dissector method for cell counting. J Neurotrauma 14: 385-398, 1997.

3. Rafols JA, Morgan R, Kallakuri S and Kreipke CW: Extent of nerve cell injury in Marmarou's model compared to other brain trauma models. Neurol Res 29: 348-355, 2007.

4. Semchenko VV, Bogolepov NN, Stepanov SS, Maksimishin SV and Khizhnyak AS: Synaptic plasticity of the neocortex of white rats with diffuse-focal brain injuries. Neurosci Behav Physiol 36: 613-618, 2006. 
5. Pozuelo-Rubio M: 14-3-3 $\zeta$ binds class III phosphatidylinositol3-kinase and inhibits autophagy. Autophagy 7: 240-242, 2011.

6. Bursch W, Hochegger K, Torok L, Marian B, Ellinger A and Hermann RS: Autophagic and apoptotic types of programmed cell death exhibit different fates of cytoskeletal filaments. J Cell Sci 113: 1189-1198, 2000.

7. Shimizu S, Kanaseki T, Mizushima N, Mizuta T, Arakawa-Kobayashi S, Thompson CB and Tsujimoto Y: Role of Bcl-2 family proteins in a non-apoptotic programmed cell death dependent on autophagy genes. Nat Cell Biol 6: 1221-1228, 2004

8. Luo CL, Li BX, Li QQ, Chen XP, Sun YX, Bao HJ, Dai DK, Shen YW, Xu HF, Ni H, et al: Autophagy is involved in traumatic brain injury-induced cell death and contributes to functional outcome deficits in mice. Neuroscience 184: 54-63, 2011.

9. Cordaro M, Impellizzeri D, Paterniti I, Bruschetta G, Siracusa R, De Stefano D, Cuzzocrea S and Esposito E: Neuroprotective effects of Co-ultraPEALut on secondary inflammatory process and autophagy involved in traumatic brain injury. J Neurotrauma, 2015.

10. Bao HJ, Zhang L, Han WC and Dai DK: Apelin-13 attenuates traumatic brain injury-induced damage by suppressing autophagy. Neurochem Res 40: 89-97, 2015.

11. Cucciolla V, Borriello A, Oliva A, Galletti P, Zappia V and Della Ragione F: Resveratrol: From basic science to the clinic. Cell Cycle 6: 2495-2510, 2007

12. Delmas D, Jannin B and Latruffe N: Resveratrol: Preventing properties against vascular alterations and ageing. Mol Nutr Food Res 49: 377-395, 2005.

13. Sakata Y,Zhuang H,Kwansa H, Koehler RC and Doré S: Resveratrol protects against experimental stroke: Putative neuroprotective role of heme oxygenase 1. Exp Neurol 224: 325-329, 2010.

14. Liu C, Shi Z, Fan L, Zhang C, Wang K and Wang B: Resveratrol improves neuron protection and functional recovery in rat model of spinal cord injury. Brain Res 1374: 100-109, 2011.

15. Saiko P, Szakmary A, Jaeger W and Szekeres T: Resveratrol and its analogs: Defense against cancer, coronary disease and neurodegenerative maladies or just a fad? Mutat Res 658: 68-94, 2008

16. Ates O, Cayli S, Altinoz E, Gurses I, Yucel N, Sener M, Kocak A and Yologlu S: Neuroprotection by resveratrol against traumatic brain injury in rats. Mol Cell Biochem 294: 137-144, 2007.

17. Gatson JW, Liu MM, Abdelfattah K, Wigginton JG, Smith S, Wolf S and Minei JP: Resveratrol decreases inflammation in the brain of mice with mild traumatic brain injury. J Trauma Acute Care Surg 74: 470-474, 2013

18. Lin CJ, Chen TH, Yang LY and Shih CM: Resveratrol protects astrocytes against traumatic brain injury through inhibiting apoptotic and autophagic cell death. Cell Death Dis 5: e1147, 2014.

19. Marmarou A, Foda MA, van den Brink W, Campbell J, Kita H and Demetriadou K: A new model of diffuse brain injury in rats. Part I: Pathophysiology and biomechanics. J Neurosurg 80: 291-300, 1994

20. Tang J, Liu J, Zhou C, Alexander JS, Nanda A, Granger DN and Zhang JH: MMP-9 deficiency enhances collagenase-induced intracerebral hemorrhage and brain injury in mutant mice. J Cereb Blood Flow Metab 24: 1133-1145, 2004.

21. Hui-guo L, Kui L, Yan-ning Z and Yong-jian X: Apocynin attenuate spatial learning deficits and oxidative responses to intermittent hypoxia. Sleep Med 11: 205-212, 2010.
22. Chen Y, Constantini S, Trembovler V, Weinstock $M$ and Shohami E: An experimental model of closed head injury in mice: Pathophysiology, histopathology and cognitive deficits. J Neurotrauma 13: 557-568, 1996.

23. Cui C, Cui Y, Gao J, Sun L, Wang Y, Wang K, Li R, Tian Y, Song S and Cui J: Neuroprotective effect of ceftriaxone in a rat model of traumatic brain injury. Neurol Sci 35: 695-700, 2014.

24. Gao X, Deng P, Xu ZC and Chen J: Moderate traumatic brain injury causes acute dendritic and synaptic degeneration in the hippocampal dentate gyrus. PLoS One 6: e24566, 2011.

25. Singleton RH, Yan HQ, Fellows-Mayle W and Dixon CE: Resveratrol attenuates behavioral impairments and reduces cortical and hippocampal loss in a rat controlled cortical impact model of traumatic brain injury. J Neurotrauma 27: 1091-1099, 2010.

26. Wang Q, Xu J, Rottinghaus GE, Simonyi A, Lubahn D, Sun GY and Sun AY: Resveratrol protects against global cerebral ischemic injury in gerbils. Brain Res 958: 439-447, 2002.

27. Sönmez U, Sönmez A, Erbil G, Tekmen I and Baykara B: Neuroprotective effects of resveratrol against traumatic brain injury in immature rats. Neurosci Lett 420: 133-137, 2007.

28. Südhof TC: The synaptic vesicle cycle: A cascade of protein-protein interactions. Nature 375: 645-653, 1995.

29. Brock TO and O'Callaghan JP: Quantitative changes in the synaptic vesicle proteins synapsin I and p38 and the astrocyte-specific protein glial fibrillary acidic protein are associated with chemical-induced injury to the rat central nervous system. J Neurosci 7: 931-942, 1987.

30. Meng H, Walker N, Su Y and Qiao X: Stargazin mutation impairs cerebellar synaptogenesis, synaptic maturation and synaptic protein distribution. Brain Res 1124: 197-207, 2006.

31. Ding JY, Kreipke CW, Schafer P, Schafer S, Speirs SL and Rafols JA: Synapse loss regulated by matrix metalloproteinases in traumatic brain injury is associated with hypoxia inducible factor-1alpha expression. Brain Res 1268: 125-134, 2009.

32. Chen P, Gu Z, Liu W and Yan Z: Glycogen synthase kinase 3 regulates $\mathrm{N}$-methyl-D-aspartate receptor channel trafficking and function in cortical neurons. Mol Pharmacol 72: 40-51, 2007.

33. Ehrlich I, Klein M, Rumpel S and Malinow R: PSD-95 is required for activity-driven synapse stabilization. Proc Natl Acad Sci USA 104: 4176-4181, 2007.

34. Ansari MA, Roberts KN and Scheff SW: A time course of contusion-induced oxidative stress and synaptic proteins in cortex in a rat model of TBI. J Neurotrauma 25: 513-526, 2008.

35. Wakade C, Sukumari-Ramesh S, Laird MD, Dhandapani KM and Vender JR: Delayed reduction in hippocampal post-synaptic density protein-95 expression temporally correlates with cognitive dysfunction following controlled cortical impact in mice. J Neurosurg 113: 1195-1201, 2010.

36. Jing CH, Wang L, Liu PP, Wu C, Ruan D and Chen G: Autophagy activation is associated with neuroprotection against apoptosis via a mitochondrial pathway in a rat model of subarachnoid hemorrhage. Neuroscience 213: 144-153, 2012.

37. Alayev A, Sun Y, Snyder RB, Berger SM, Yu JJ and Holz MK: Resveratrol prevents rapamycin-induced upregulation of autophagy and selectively induces apoptosis in TSC2-deficient cells. Cell Cycle 13: 371-382, 2014. 